\title{
Urinary carboxylic acids (UCAs) in subjects with autism spectrum disorder and their association with bacterial overgrowth
}

https://doi.org/10.1515/revac-2020-0109

Received May 21, 2020; accepted July 27, 2020

\begin{abstract}
In this study, the levels of concentration of carboxylic acids (benzoic acid, $p$-hydroxybenzoic acid, $p$-hydroxyphenylacetic acid, and hippuric acid) in the urine of autistic children were investigated and compared. The increased excretion of carboxylic acids is related to excessive bacterial activity in the gut, called bacterial overgrowth, which has been related to autism spectrum disorder (ASD) as an impairment in the gut-brain axis. The investigation was based on the analysis of urine samples obtained from 120 ASD children. To identify and quantify urinary carboxylic acids (UCAs), we applied gas chromatography coupled with mass spectrometry (GCMS). Additionally, we checked the influence of probiotic supplementation, gender, body mass index (BMI) value and age of children on the level of different selected compounds. Most of the obtained results were found within reference ranges. In some cases, the levels of benzoic acid, $p$-hydroxybenzoic acid, and $p$-hydroxyphenylacetic acid were particularly elevated. Statistically significant differences were observed in supplementation with probiotics and the level of $p$-hydroxyphenylacetic acid $(p=0.036)$. The obtained results may indicate disturbances in the intestinal flora in some autistic patients and suggest that supplements may have an influence on the levels of
\end{abstract}

\footnotetext{
*Corresponding author: Paulina Gątarek, Institute of General and Ecological Chemistry, Faculty of Chemistry, Lodz University of Technology, 116 Zeromskiego Street, 90-924 Lodz, Poland, e-mail: gatarekpaulina@gmail.com, Tel.:+48 42 631-31-14

${ }^{1}$ Institute of General and Ecological Chemistry, Faculty of Chemistry, Lodz University of Technology, 116 Zeromskiego Street, 90-924 Lodz, Poland

${ }^{2}$ Council for Nutritional and Environmental Medicine, Mo i Rana, Norway

${ }^{3}$ Department of Neurological and Movement Sciences, University of Verona, Verona, Italy
}

Ә Open Access. ( 2020 Paulina Gątarek, published by De Gruyter. @ @ Attribution 4.0 Public License. carboxylic acids in urine. Due to the small population of children taking the supplement, further study are needed.

Keywords: ASD, autism, carboxylic acids, bacterial overgrowth, probiotic supplementation.

\section{Introduction}

Both the outside world and the internal humanenvironment immunologically interface in the gastrointestinal tract (GIT). A bidirectional interaction between brain and gut enables humans to finely modulate not only metabolism and bio-energetics but also a huge number of foreign substances entering the organism via the GIT, raising formidable concerns about immunity, tolerance, the same concept of a biological "self" and cognition awareness [1-4]. Along with being responsible for digesting and absorbing nutrients, GIT is also exposed to dietary antigens, bacterial products, and viable microorganisms. Its complexity cannot be insulated from the whole perception of an organic self, i.e., from the immune and neural control and in this regard, the new concept of brain-gut axis should enlighten many complex issues regarding neurological pathologies such as autism spectrum disorder (ASD) [5-7]. The role of microbiome (also known as microbiota) is of major importance in the modulation of gut immunity and the general host's immune response [8,9]. Along with the host's immune system, microbiota plays an essential role in the proper functioning of the body participating in metabolic tracts and acting as a source of key nutrients. In this sense, the intestinal microbial imbalance can be a consequence of the exposure to many environmental factors, including further microorganisms, xenobiotics, and pollutants. Alterations in the microbiota and the presence of enteric pathogens may contribute to microbial dysbiosis, with possible suggestions on ASD pathogenesis [10-13]. The presence of specific metabolites from microorganisms 
and pathogenic toxins associated with the microflora overgrowth in the gut is among the major symptoms of this pathology. Intestinal dysbiosis is usually a long-term condition in which the accumulated toxic compounds can significantly affect the functions of many different organs [14-16]. Several studies have shown that some species of bacteria can degrade various amino acids such as tyrosine. For example, Clostridium difficile and Proteus vulgaris play a role in the transamination of tyrosine, which leads to the formation of $p$-hydroxyphenylacetate. $C$. difficile is responsible for the decarboxylation of $p$-hydroxyphenylacetate to $p$-cresol. Elevated levels of $p$-hydroxyphenylacetate are seen in various disorders, including organ failure and abnormal bowel movements, which are directly related to intestinal dysbiosis. Thus, $p$-hydroxyphenylacetate is a great example of a compound that serves as a biomarker of excess microbial activity in the GIT $[14,17]$. Further compounds of microbial origin enter the liver detoxification pathways and are then excreted in a modified form, in which they too can serve as biomarkers for bacterial overgrowth. Piperidine, which is a well-known product of the conversion of bacterial amines, is used as a sensitive indicator of changes in the gastrointestinal microflora in celiac disease [14]. Additionally, some genetic pre-dispositions and polymorphism determine the way how substances of the bacterial origin are metabolized, providing indicators for assessing microbial balance [18]. In people with a relatively common genetic defect in fatty acid oxidation, 3-phenylpropionate (an anaerobic bacterial product) is converted into 3-phenylpropionylglycine, whereas in healthy individuals it is converted to hippuric acid [14]. The aminoacidic balance of systemic metabolism is partly due to the correct modulation of the gut microbiome.

Other compounds associated with intestinal dysbiosis are dicarboxylic acids (DCAs). The analysis of DCAs in the urine is an important tool in the diagnosis of metabolic disorders. DCAs provide valuable information about metabolism and potential problems linked to intestinal dysbiosis and energy production. These acids include adipic, succinic and suberic acids. In the literature, disturbances of DCAs levels in autistic children have been reported, but the reason for these disturbances in autism is not clear yet $[19,20]$. Research conducted by Kałużna-Czaplińska et al. (2011) suggested that vitamin $B$ supplementation is effective in decreasing the level of DCAs in the urine of children with ASD. Researchers examined the levels of adipic, succinic and suberic acids in the urine of 30 children with ASD before and after supplementation, taken twice a day for 3 months (magnesium lactate, daily dose $200 \mathrm{mg}$; vitamin B6, daily dose 500mg; and vitamin B2, daily dose 20mg), and 10 nonautistic children. They observed a significantly higher level of adipic acid in children with ASD compared to nonautistic children. Considering vitamin B supplementation, they observed that in the urine of ASD children before supplementation the levels of adipic, succinic, and suberic acids were significantly higher compared to children after supplementation [19].

In the next research conducted by Puig-Alcaraz et al. (2016) suggested no increase in the level of urinary adipic acid in ASD children compared to typicallydeveloping children, who were a control group. However, a statistically significant difference between the increase in the level of adipic acid in autistic children without vitamin B supplementation compared with ASD children with supplementation or control group were observed. No significant difference in the level of suberic acid was observed. Moreover, no significant correlation between the level of adipic acid and the duration of vitamin B supplementation was observed. The authors also investigated the relationship between the level of adipic acid and the score symptoms. They suggested an association between elevated adipic acid levels in urine ASD children and reduced core ASD symptom severity. The authors have shown that in children with ASD, an increase in the level of adipic acid was correlated with the severity of the deficit in communication skills and socialization [20].

The reason for the disturbances of DCAs is not known. The literature suggested that increased succinate excretion is characteristic of CoQ10 and riboflavin deficiencies. Increased excretion of succinic acid is caused by the deficit of riboflavin [19-22]. Adipic and suberic acids are produced by the omega $(\omega)$-oxidation pathway. The levels of adipic and suberic acids may increase due to a rise in $\omega$-oxidation, which may be caused by impaired $\beta$-oxidation of fatty acids in ASD. Altered mitochondrial metabolism may increase the activity of omega fatty acid oxidation, which then leads to increased production of adipic and suberic acid [20].

Tyrosine can also be converted into $p$-cresol and methyl $p$-hydroxybenzoate via the metabolic processes of bacteria and protozoa. These compounds are not produced in a properly functioning human body. It should be highlighted that cresol is highly toxic, and elevated levels of cresol secretion are characteristic of intestinal bacterial overgrowth caused by $C$. difficile. Cresol inhibits the enzymatic activity of dopamine beta-hydroxylase, leading to an imbalance of neurotransmitters. To reduce the level of this compound, oral absorbents are used, including activated carbon. $p$-hydroxybenzoate can be 
produced from food products containing parabens such as preservatives; moreover, it can be formed from glucose by Escherichia coli [14,23].

Bacterial phenylalanine deamination leads to the formation of benzoate, which together with glycine forms hippuric acid in the liver. Benzoic acid is used in foods as a preservative; thus, consuming specific foods can also lead to elevated levels of hippuric acid in urine. Elevated levels of benzoate indicate a shortage of glycine or pantothenic acid (vitamin B5) because these compounds are necessary for its transformation into a hippurate [14,24]. The presence of hippuric acid in urine may also result from the decomposition of chlorogenic acid by bacteria. This acid is found in many fruits, vegetables and beverages [23]. Any of these catabolic markers can be used as a probing molecule to follow ASD pathology, at least theoretically.

Catechins present in green and black tea are converted into 3-phenylpropionate by intestinal bacteria. Then, this absorbed compound is oxidized to benzoate and converted into a hippurate by human intestinal and liver enzymes. Increased growth of a bacterial population also results in an elevated concentration of excreted hippurate [25]. Furthermore, D-lactic acid is a major metabolite of several strains of bacteria that are present in the human gut; a wide range of bacteria can convert simple sugars into this compound. Moreover, human tissues may produce trace concentrations of D-lactic acid. The formation of D-lactic acid is also connected with a growing population of Lactobacillus acidophilus in the intestine. This increase occurs under acidic conditions, i.e., when the rate of organic acid formation is sufficiently high to be suitable for bacterial growth. In the liver, D-lactate can be converted into carbon dioxide, yet only to a small extent, as opposed to L-lactate, which is metabolized in skeletal muscle and tissues [14]. Lactic acidosis occurs with elevated levels of $p$-hydroxyphenylacetate, $p$-hydroxyphenylbenzoate and hippurate, which are also derived from bacterial metabolism and intestinal infection [26].

Another major catabolite is D-arabinitol. D-Arabinitol (DA), five-carbon sugar alcohol, is produced by most pathogenic Candida species, which are of great clinical importance due to their transmission by direct invasion of the GIT and their ability to overwhelm immune responses. A great number of Candida species grow best on carbohydrate substrates. When the organism is grown on arabinose, aldose reductase and xylitol dehydrogenase activities are induced. DA is indicative of arabinitol in the body and is proportional to the arabinitol/creatinine ratio in urine or serum. Measuring serum DA allows the diagnosis of invasive candidiasis. Immunocompromised patients suffering from invasive candidiasis have been observed to have an elevated $\mathrm{DA} /$ creatinine ratio in urine. Additionally, an elevated DA/L-arabinitol ratio in urine has been described to be a sensitive diagnostic biomarker for invasive candidiasis in infants. Probiotics can be effective in reducing DA levels in the urine of autistic children [14,24,27].

The main aim of the paper was to compare the levels of organic acids connected with bacterial overgrowth in autistic children. Several children received probiotic supplementation whose influence on the levels of the determined acids were examined. Additionally, the investigation of whether the concentration is related to gender, age, or BMI was performed.

\section{Results and discussion}

In the present study, a simple, precise, and sensitive GC-MS method for the determination of carboxylic acids in urine samples was applied. Table 1 shows the values found for urinary carboxylic acids (benzoic acid, hippuric acid, $p$-hydroxybenzoic acid, and $p$-hydroxyphenylacetic acid) in the study population. The results were calculated as a ratio of the analyte of interest and urinary creatinine concentration in the unit $\mu \mathrm{g} / \mathrm{mg}$ creatinine $(\mu \mathrm{g} / \mathrm{mg} \mathrm{C})$. The median and mean value for all determined organic acids fall within the reference ranges. The obtained results were characterized by high variability. Table 2 shows the number of patients and median for values below, within and above the reference range which is available online at The Human Metabolome Database [28,29].

The levels of acids were increased in: $n=9$ for benzoic acid (median: $17.53 \mathrm{mmol} / \mathrm{mmol}$ Creatinine), $n=17$ for p-hydroxybenzoic acid (median: $16.68 \mathrm{mmol} / \mathrm{mmol}$ Creatinine), and $n=11$ for $p$-hydroxyphenylacetic acid (median: $53.82 \mathrm{mmol} / \mathrm{mmol}$ Creatinine). Simultaneously, the concentration of $p$-hydroxyphenylacetic acid was decreased in $n=10$ (median: $2.42 \mathrm{mmol} / \mathrm{mmol}$ Creatinine). All the results obtained for hippuric acid were within the reference range.

Application of the Shapiro-Wilk test showed that the hypothesis that the data was normally distributed could be rejected [ $p<0.05]$. The levels of carboxylic acids were compared between the groups (age, gender, probiotic supplementation, and BMI) using Mann-Whitney U test. Individual differences in the levels of carboxylic acids between the groups were found. Considering the difference in the $p$-value of $<0.05$ to be statistically significant, the BMI valueturned out to have an impact on thelevel of onlybenzoic acid ( $\mathrm{p}=0.001$ ). Furthermore, among the studied population, $81.6 \%$ were underweight, $15 \%$ had normal weight, and 3.4 
Table 1 Values obtained for quantification of determined acids ( $\mu \mathrm{mol} / \mathrm{mmol}$ of creatinine) in urine samples of all tested population of ASD children

\begin{tabular}{lllllllll}
\hline Name of the acid & reference range $^{*}$ & mean & median & $\min$ & $\max$ & LQ & UQ & SD \\
\hline benzoic acid & $<10.3$ & 4.5 & 2.8 & 0.1 & 39.3 & 1.5 & 5.3 & 5.6 \\
\hline hippuric acid & $<969.8$ & 97.2 & 69.1 & 3.2 & 906.9 & 26.8 & 116.9 & 116.2 \\
\hline p-hydroxybenzoic acid & $0.1-11.1$ & 8.6 & 3.6 & 0.2 & 302.0 & 2.1 & 7.8 & 28.0 \\
\hline p-hydroxyphenylacetic acid & $4.9-30.6$ & 20.4 & 14.5 & 0.5 & 147.7 & 8.8 & 25.5 & 20.1 \\
\hline
\end{tabular}

LQ- lower quartile, UQ- upper quartile, SD- standard deviation, *- reference range available online at The Human Metabolome Database.

Table 2 Number of patients and median for values below, within and above the reference range

\begin{tabular}{|c|c|c|c|c|c|c|}
\hline & $\begin{array}{l}n \text { below the } \\
\text { reference range }\end{array}$ & median & $\begin{array}{l}n \text { within the } \\
\text { reference range }\end{array}$ & median & $\begin{array}{l}n \text { above the } \\
\text { reference range }\end{array}$ & median \\
\hline benzoic acid & - & - & 111 & 2.5 & 9 & 17.5 \\
\hline hippuric acid & - & - & 120 & 69.1 & - & - \\
\hline p-hydroxybenzoic acid & - & - & 103 & 2.9 & 17 & 16.7 \\
\hline p-hydroxyphenylacetic acid & 10 & 2.4 & 99 & 14.5 & 11 & 53.8 \\
\hline
\end{tabular}

Figure 1 Relationship between BMI values and the concentrations of the analyzed carboxylic acids
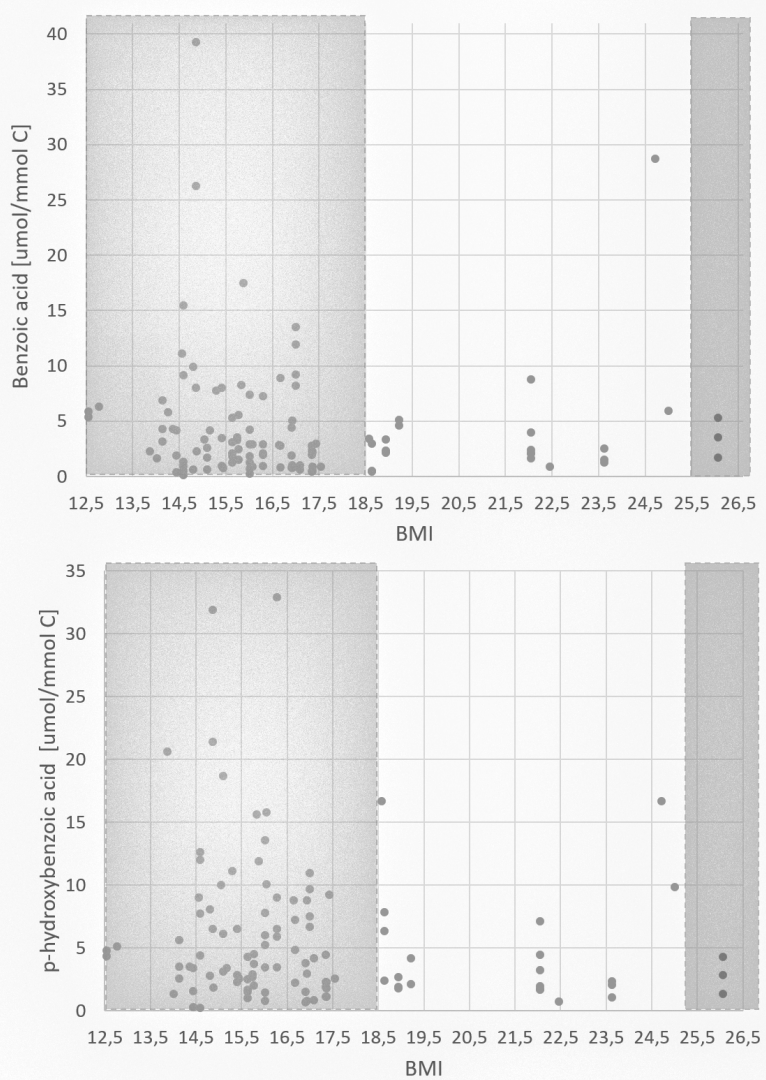

$\mathrm{BM}$

$\%$ were overweight. Relationship between BMI values and the concentrations of the analyzed carboxylic acids shows Figure 1. No link between the age value and the levels of the acids was found ( $p>0.05$ ). Moreover, application of a MannWhitney $\mathrm{U}$ test showed a difference between the levels of benzoic acid in autistic boys and girls $(\mathrm{p}=0.04)$. The gender

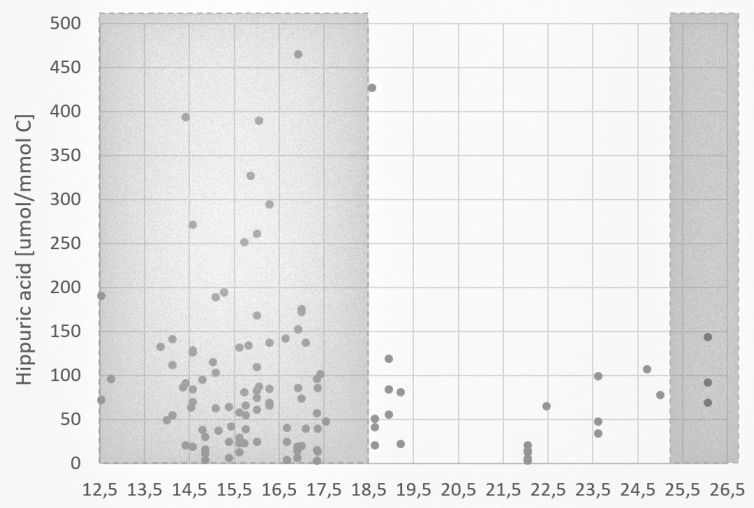

$\mathrm{BMI}$

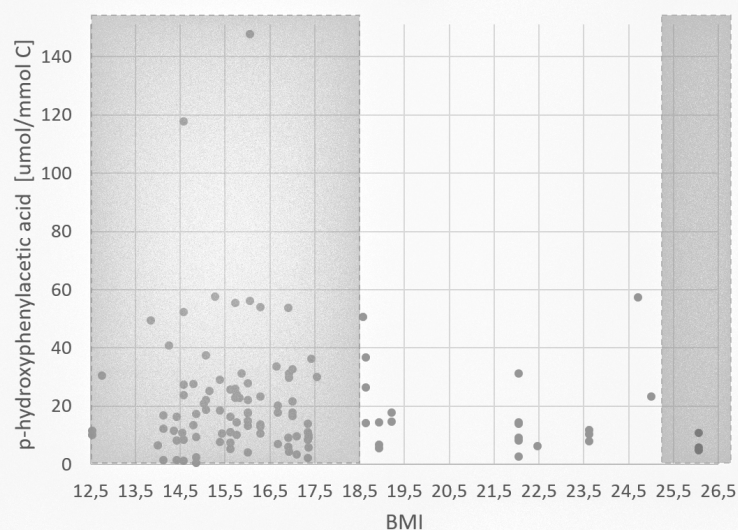

of patients does not seem to influence the concentrations of the other acids (hippuric acid: $\mathrm{p}=0.22 ; p$-hydroxybenzoic acid: $\mathrm{p}=0.74 ; p$-hydroxyphenylacetic acid: $\mathrm{p}=0.25$ ). Figure 2 presents distribution of obtained results in both groups: boys and girls.

A small group of ASD children received 
Figure 2 Box and Whisker plots for determining acids level in groups: boys and girls. In these box plots, medians inside the $25-75 \%$ interquartile range (IQR) are presented
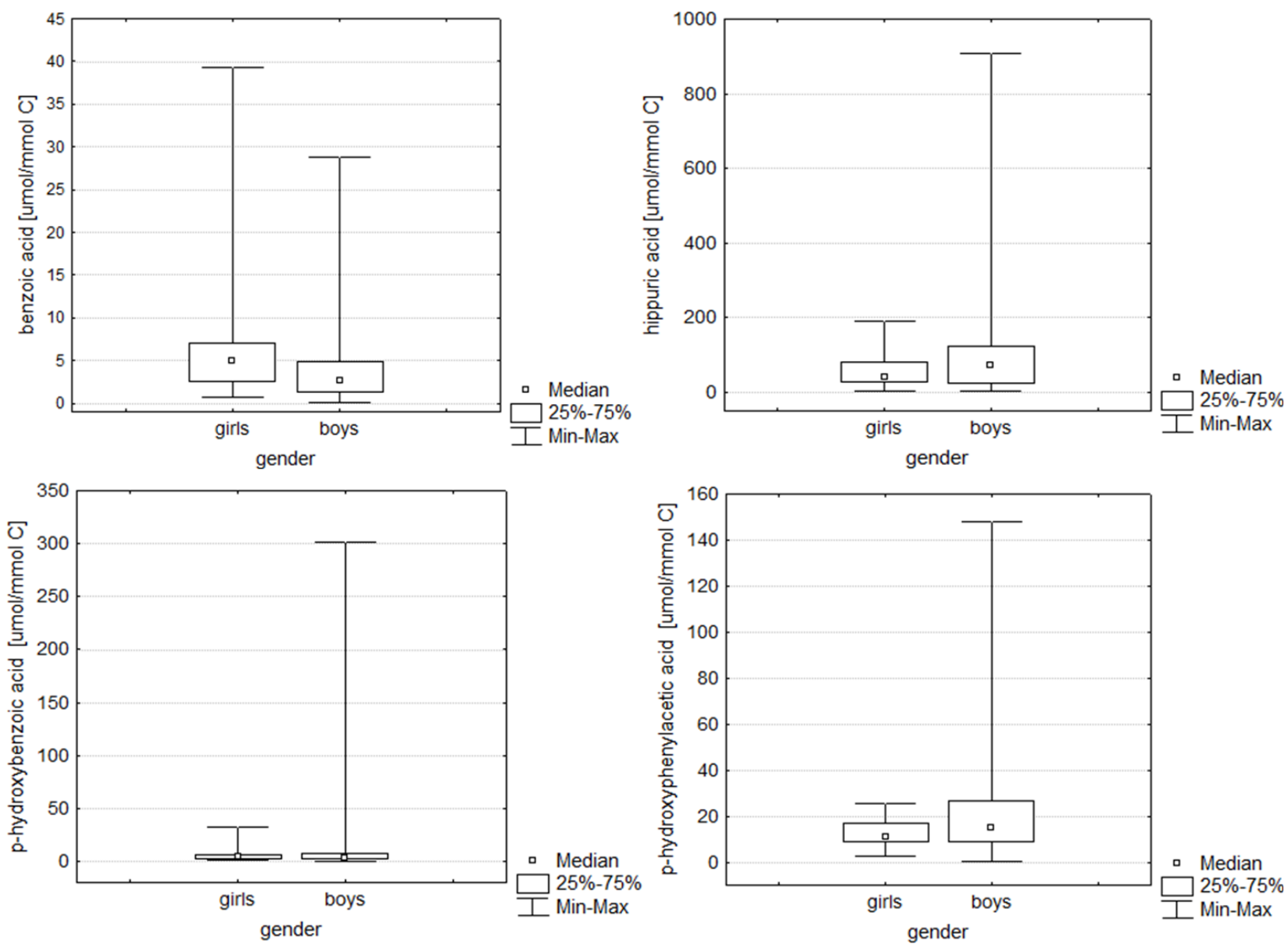

Table 3 Values obtained for quantification of determined acids ( $\mu \mathrm{mol} / \mathrm{mmol}$ of creatinine) in urine samples of all tested population of ASD children with and without probiotic supplementation

\begin{tabular}{lllllllll}
\hline name of acid & supplementation & mean & median & $\min$ & $\max$ & LQ & UQ & SD \\
\hline benzoic acid & yes & 4.5 & 3.3 & 0.5 & 28.8 & 2.3 & 4.2 & 5.9 \\
\cline { 2 - 9 } & no & 4.5 & 2.6 & 0.1 & 39.3 & 1.3 & 5.3 & 5.7 \\
\hline \multirow{2}{*}{ hippuric acid } & yes & 110.1 & 77.2 & 6.1 & 427.8 & 47.2 & 115.1 & 108.5 \\
\cline { 2 - 9 } & no & 94.5 & 65.8 & 3.2 & 906.9 & 23.1 & 118.6 & 118.1 \\
\hline p-hydroxybenzoic acid & yes & 7.6 & 4.5 & 1.9 & 20.64 & 2.8 & 10.0 & 6.0 \\
\cline { 2 - 9 } & no & 8.8 & 3.5 & 0.2 & 302.0 & 1.8 & 7.2 & 30.7 \\
\hline $\begin{array}{l}\text { p-hydroxyphenylacetic } \\
\text { acid }\end{array}$ & yes & 29.4 & 22.1 & 7.7 & 147.7 & 11.1 & 30.1 & 30.7 \\
\cline { 2 - 9 } & no & 18.5 & 14.2 & 0.5 & 117.7 & 8.3 & 24.0 & 16.6 \\
\hline
\end{tabular}

LQ- lower quartile, UQ- upper quartile, SD- standard deviation

supplementation with probiotics $(n=21)$. The influence of their application was presented in Table 3. The median and mean values were similar in both groups and were within reference ranges. It should be highlighted that in most children receiving supplementation no abnormal values were found for the determined acids, only one child had a higher level of benzoic acid in urine.
Figure 3 presents distribution of obtained results in the groups with and without probiotic supplementation. Individual differences in the levels of carboxylic acids between the two groups (with and without probiotic supplementation) were found. Considering the difference in the $p$-value of $<0.05$ to be statistically significant, the supplementation with probiotics turned out to have 
an impact on the level of only $p$-hydroxyphenylacetic acid $(\mathrm{p}=0.036)$. However, the result may be related to the small population receiving the supplements $(\mathrm{n}=21)$. Further research on a larger group of autistic patients in equinumerous groups could describe the effect of probiotics on the levels of these carboxylic acids, which are biomarkers of bacterial overgrowth.

Finally, the statistical analysis showed a strong positive correlation between the concentration of $p$-hydroxybenzoic acid and hippuric acid (correlation value 0.71), a weaker correlation between $p$-hydroxyphenylacetic acid and hippuric acid (correlation value 0.45 ), and a very weak positive correlation between $p$-hydroxyphenylacetic acid and $p$-hydroxybenzoic acid (correlation value 0.26 ).

Typical GC-MS total ion chromatograms (TIC) (from 8 to $18 \mathrm{~min}$ ) of urine samples obtained from autistic children are presented in Figure 4.

The Urisys 1100 system enabled us to assess the condition of the patients' urinary tracts. Abnormalities relating to three urine parameters, specific gravity $(\mathrm{n}=10), \mathrm{pH}(\mathrm{n}=5)$, nitrites $(\mathrm{n}=2)$ and ketones $(\mathrm{n}=4)$ were identified in a few cases, while other basic parameters were within normal ranges. The determination of basic urine parameters showed that the patients did not suffer from urinary tract-related disorders and that the results obtained from the chromatography-based techniques were reliable. The possibility of obtaining the results that could be falsified because of the urinary tract dysfunction was therefore eliminated.

The assessed levels of the analyzed acids were compared to the reference values, which are available online at The Human Metabolome Database [28,29]. Reference ranges for hippuric acid and benzoic acid were established by BC Children's Hospital Biochemical Genetic Lab by GC-MS using urine samples obtained from children 1-18 years of age. Current literature reports that the urine concentration of hippuric acid should not exceed $969.8 \mu \mathrm{mol} / \mathrm{mmol}$ creatinine. Our study showed that none of the samples exceeded these values. In a few cases, the concentrations of the other acids were exceeded. Simultaneously, the level of $p$-hydroxyphenylacetic acid was decreased in a few children. In the studied population of autistic children, a small group received probiotics $(\mathrm{n}=21)$. These supplements are described as a culture of living microorganisms that improve the properties of the bacterial GIT microflora [14]. Literature reports that supplementation with probiotics, which eliminates characteristic symptoms, is an effective treatment for irritable bowel syndrome [30]. The ability of probiotics to protect the natural microflora is of scientific interest and has been previously described in detail [31]. The influence of these supplements on the gut microflora could have an impact on the levels of carboxylic acids in urine. This assumption was verified, was found statistically significant on the level of only $p$-hydroxyphenylacetic acid. Due to the small population of children taking the supplement, further study are needed.

In the literature, the relationship between imbalanced microflora and the presence of chronic gastrological symptoms is widely described. Fast and visible clinical improvement after treatment with vancomycin, metronidazole, and the recurrence of characteristic symptoms after interruption of these medications confirm this dependency [32,33]. Gastrointestinal problems, including bloating, abdominal pain, diarrhea and gastroesophageal reflux disease, may be reflected in the BMI values of children who suffer from these symptoms. The performed study showed influence of the BMI value on the level of benzoic acid.

A number of reports have described a link between ASD and various diseases connected with the digestive system, including those related to the functioning of the gut $[33,34]$. It has been suggested that certain changes in the gut may contribute to the increased intestinal permeability of exogenous peptides, which in turn adversely affects brain development, potentially causing the neurological disorders that are responsible for autism symptoms [35]. Scientists do not have a clear answer concerning the causes of the gastrointestinal disorders and intestinal abnormalities seen in autistic children; genetic and environmental factors, including intestinal microflora irregularities, have been considered. Some patients diagnosed with autism are subject to recurring antibiotic therapy, which can contribute to intestinal dysbiosis by affecting the stability of the intestinal microflora [36]. The effect of microorganisms on autistic behaviors has been confirmed, yet no specific group of bacteria has been directly indicated [32,37]. Moreover, there is a possibility that the key organism responsible for these disturbances may be present in small, even trace quantities. Therefore, the most effective method to diagnose such disorders is measuring substances/metabolites (biomarkers) produced by intestinal microorganisms and a further description of their influence on the human body [37].

Undertaking appropriate steps and interventions may significantly affect improvement in autistic behavior. The introduction of an appropriate diet, excluding specific products, e.g., peptides, which are believed to affect brain function by crossing the gut-blood and then blood-brain barriers, is recommended by some specialists [35,38]. The application of such a diet was reported to improve language skills, social interactions and the ability to 
Figure 3 Box and Whisker plots for the determined acid level in the groups with and without probiotic supplementation. In these box plots, medians inside the $25-75 \%$ interquartile range (IQR) are presented
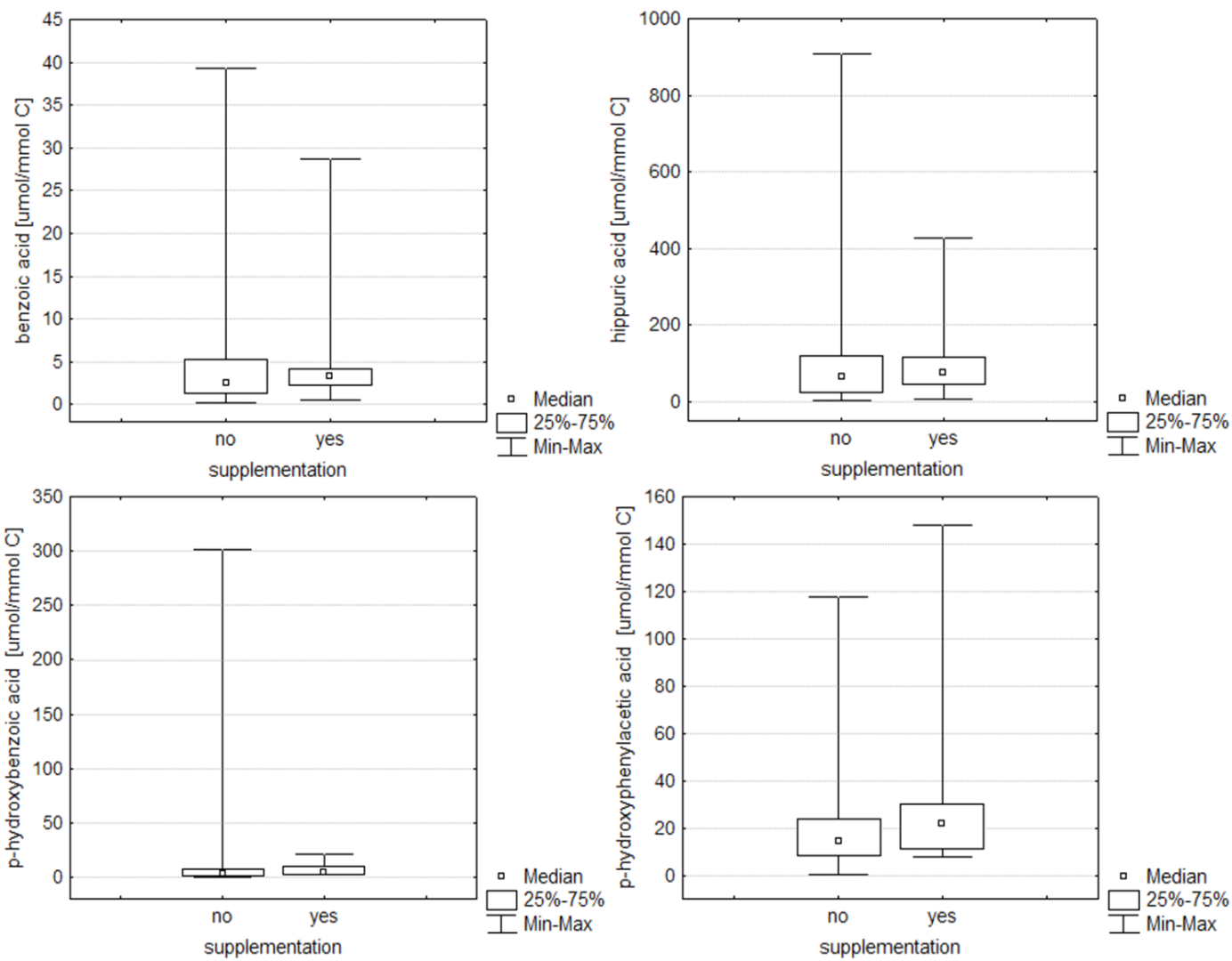

Figure 4 The typical total ion chromatogram of derivatized organic acids in the urine of autistic children. 1- benzoic acid; 2 p-hydroxybenzoic acid; 3- $p$-hydroxyphenylacetic acid; 4- hippuric acid; IS- internal standard (heptadecanoic acid)

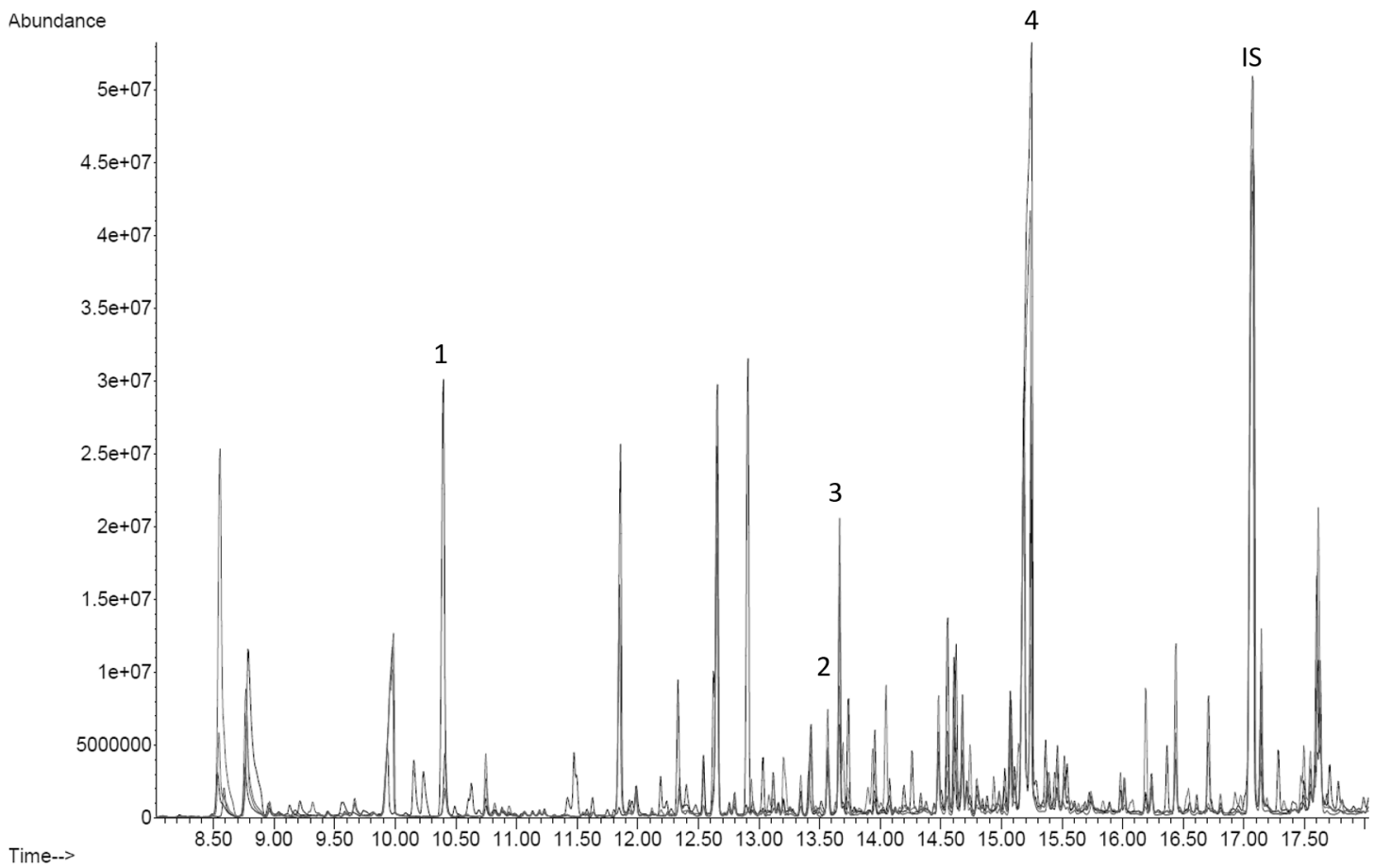


concentrate [31,35]. Diets may also have a positive effect on BMI, which can result in decreased levels of some organic acids in urine.

\section{Conclusion}

Our research was based on the determination of four compounds associated with the activity of intestinal bacteria. In a few cases the levels of benzoic acid, $p$-hydroxybenzoic acid, and $p$-hydroxyphenylacetic acid were elevated. Additionally, the concentration of $p$-hydroxyphenylacetic acid was decreased in several patients. These abnormalities in urinary organic acids excretion may indicate disturbances in the intestinal flora in some of the autistic patients, which may suggest some metabolic or dietary abnormalities in autism. Futurestudies on a larger cohort focused on the intestinal bacterial flora of ASD patients will improve our understanding of the mechanisms of autism; more broadly define the nature of the abnormality, define the relationship between gut flora and ASD, and help develop new therapeutic interventions. This could result in simplifying the diagnosis of autism and introducing a more individualized approach to therapy.

\section{Experimental}

\subsection{Patients and sample collection}

This study was restricted to children with an ASD diagnosis in compliance with the criteria detailed in The International Classification of Diseases [39]. Overnight urine samples were collected from 120 autistic children (3-18 years of age) who underwent rehabilitation in the Center for Diagnosis and Therapy of Autism in Łódź

Table 4 Stratification of the tested population

\begin{tabular}{llll}
\hline number of analyzed samples & $\mathrm{n}=120$ & boys & $\mathrm{n}=108$ \\
\cline { 3 - 3 } age ranges & girls & $\mathrm{n}=12$ \\
\hline $\begin{array}{l}\text { number of children with probiotic } \\
\text { supplementation }\end{array}$ & $\mathrm{n}$ of age & \\
\hline $\begin{array}{l}\text { number of children without } \\
\text { probiotic supplementation }\end{array}$ & $\mathrm{n}=99$ \\
\hline number of underweight children & $\mathrm{n}=98$ & \\
\hline $\begin{array}{l}\text { number of children of normal } \\
\text { weight }\end{array}$ & $\mathrm{n}=18$ \\
\hline number of children of overweight & $\mathrm{n}=4$ \\
\hline number of obese children & - \\
\hline
\end{tabular}

Navicula (Poland) and were stored at $-20^{\circ} \mathrm{C}$ until analysis. All autistic children were assessed and diagnosed by clinicians specializing in the diagnosis and management of ASD children from the Center. No children were on a gluten-free, casein-free or sugar-free diet. A small cohort of the subjects underwent supplementation with probiotics $(n=21)$. The study was conducted in accordance with the Declaration of Helsinki, and the protocol was approved by the Ethics Committee of the Polish Mother's Memorial Hospital Research Institute of 30/2018.

BMI was calculated for all patients by dividing weight in kilograms by the square of height in meters. Based on BMI, subjects are classified in four classes: underweight (BMI $\left.<18.5 \mathrm{~kg} / \mathrm{m}^{2}\right)$, normal weight $\left(18.5 \mathrm{~kg} / \mathrm{m}^{2} \leq \mathrm{BMI} \leq 24.9\right.$ $\left.\mathrm{kg} / \mathrm{m}^{2}\right)$, overweight $\left(25.0 \mathrm{~kg} / \mathrm{m}^{2} \leq \mathrm{BMI} \leq 29.9 \mathrm{~kg} / \mathrm{m} 2\right)$ and obese (BMI $\geq 30.0 \mathrm{~kg} / \mathrm{m}^{2}$ ). The specific characteristics of the studied population are presented in Table 4.

\subsection{Sample preparation and analytical methods}

Urine samples were thoroughly mixed in order to maintain homogeneity and aliquoted. Urine was collected into 1.5 $\mathrm{mL}$ Eppendorf tubes and stored at $-20{ }^{\circ} \mathrm{C}$ until GC-MS analysis. For sample preparation, a simple modification of the method described by Zhang et al. was used [40].

Urine samples were thawed at room temperature. Acids were extracted using ethyl acetate and diethyl ether and derivatized with BSTFA (N,O-bis[trimethylsilyl] trifluoroacetamide). Onto the analytical column $1 \mu \mathrm{l}$ of supernatant was injected into an Agilent 6890N Network GC system and a 5973 Network Mass Selective equipped with a capillary column (J\&W Ultra Inert HP-5ms; Agilent Technology, Santa Clara, CA, USA; $30 \mathrm{~m} \times 0.25 \mathrm{~mm}$ internal diameter; film thickness, $0.25 \mu \mathrm{m}$ ). The injector temperature was set at $250{ }^{\circ} \mathrm{C}$. Helium was used as a carrier gas at a constant flow rate of $0.9 \mathrm{mLmin}^{-1}$ through the column. The column temperature was initially kept at $75^{\circ} \mathrm{C}$ for $5 \mathrm{~min}$ and then increased to $280^{\circ} \mathrm{C}$ at $15^{\circ} \mathrm{Cmin}^{-1}$. The MS quadrupole temperature was set at $150^{\circ} \mathrm{C}$ and the ion source temperature at $230{ }^{\circ} \mathrm{C}$. Masses were acquired from m/z 50-550. Mass Hunter Workstation Software was used to identify and quantify compounds. Results were expressed as ratios to the urinary creatinine concentration in $\mu \mathrm{mol} / \mathrm{mmol}$ creatinine. Additionally, samples were examined with a reflectance photometer Urisys 1100 Urine Analyzer (Roche, Basel, Switzerland), which enables the measurement of basic urine parameters (specific gravity, $\mathrm{pH}$, leukocyte, nitrite, protein, glucose, ketone, urobilinogen, bilirubin, erythrocyte excretion). 


\subsection{Urinary Creatinine Determination}

The level of the modified carboxylic acids in urine was standardized by conversion to the creatinine level. Creatinine level was investigated by using the highperformance liquid chromatography method reported elsewhere [41,42].

\subsection{Statistical analysis}

After the GC-MS analysis, each sample was represented by a GC-MS total ion chromatogram and the peak areas of compounds were integrated. The peak area ratio of each compound to the corresponding internal standard was calculated as the response. The majority of the peaks in the chromatograms were identified as endogenous metabolites by the NIST mass spectra library.

Data were statistically evaluated using the statistical analysis package (StatSoft, Polska STATISTICA, version 9.0., Quest Software, Aliso Viejo, CA, USA). All variables were checked for normal distribution of the results by the Shapiro-Wilk's test. The nonparametric Mann-Whitney U test was used, as appropriate, to compare the concentration of carboxylic acids between the groups. All comparisons were two-sided with a p-value of less than 0.05 used to indicate statistical significance. Subsequently, correlation analysis was carried out to discover possible relations among the variables.

\section{Acknowledgments}

We would like to thank Dr. Anna Rozetti-Szymańska for medical consultation and the parents of autistic children from the Center for Diagnosis and Therapy of Autism, Lodz, Poland for their help in collecting urine samples from autistic children.

Conflicts of Interest: The authors declare no conflict of interest.

\section{References}

[1] Kim N, Yun M, Oh YJ, Choi HJ. Mind-altering with the gut: modulation of the gut-brain axis with probiotics. J Microbiol. 2018;56(3):172-82.

[2] Carabotti M, Scirocco A, Maselli MA, Severi C. The gut-brain axis: interactions between enteric microbiota, central and enteric nervous systems. Ann Gastroenterol. 2015;28(2):203-9.
[3] Petta I, Fraussen J, Somers V, Kleinewietfeld M. Interrelation of Diet, Gut Microbiome, and Autoantibody Production. Front Immunol. 2018;9:439.

[4] Stefano GB, Ptacek R, Raboch J, Kream RM. Microbiome: A Potential Component in the Origin of Mental Disorders. Med Sci Monit. 2017;23:3039-43.

[5] Kraneveld AD, Szklany K, de Theije CG, Garssen J. Gut-to-Brain Axis in Autism Spectrum Disorders: Central Role for the Microbiome. Int Rev Neurobiol. 2016;131:263-87.

[6] Rosenfeld CS. Microbiome Disturbances and Autism Spectrum Disorders. Drug Metab Dispos. 2015;43(10):1557-71.

[7] Vuong HE, Hsiao EY. Emerging Roles for the Gut Microbiome in Autism Spectrum Disorder. Biol Psychiatry. 2017;81(5):411-23.

[8] Kumar H, Bot A. In this issue: effect of gut microbiome on mucosal immunity and enteric diseases. Int Rev Immunol. 2018;37(2):77-8.

[9] Shi N, Li N, Duan X, Niu H. Interaction between the gut microbiome and mucosal immune system. Mil Med Res. 2017;4:14.

[10] Lim JS, Lim MY, Choi Y, Ko G. Modeling environmental risk factors of autism in mice induces IBD-related gut microbial dysbiosis and hyperserotonemia. Mol Brain. 2017;10(1):14.

[11] Liu X, Cao S, Zhang X. Modulation of Gut Microbiota-Brain Axis by Probiotics, Prebiotics, and Diet. J Agric Food Chem. 2015;63(36):7885-95.

[12] Doenyas C. Gut Microbiota, Inflammation, and Probiotics on Neural Development in Autism Spectrum Disorder. Neuroscience. 2018;374:271-86.

[13] Bruce-Keller AJ, Salbaum JM, Berthoud HR. Harnessing Gut Microbes for Mental Health: Getting From Here to There. Biol Psychiatry. 2018;83(3):214-23.

[14] Carding S, Verbeke K, Vipond DT, Corfe BM, Owen LJ. Dysbiosis of the gut microbiota in disease. Microb Ecol Health Dis. 2015;26:26191.

[15] Pulikkan J, Maji A, Dhakan DB, Saxena R, Mohan B, Anto MM, et al. Gut Microbial Dysbiosis in Indian Children with Autism Spectrum Disorders. Microb Ecol. 2018;76(4):1102-14.

[16] Lyu Y, Wu L, Wang F, Shen X, Lin D. Carotenoid supplementation and retinoic acid in immunoglobulin $A$ regulation of the gut microbiota dysbiosis. Exp Biol Med (Maywood). 2018;243(7):613-20.

[17] Dawson LF, Stabler RA, Wren BW. Assessing the role of p-cresol tolerance in Clostridium difficile. J Med Microbiol. 2008;57:745-9.

[18] Walker MM. Inflammation, Genetics, Dysbiosis, and the Environment: New Paradigms for Diagnosis in Complex Chronic Gut Syndromes. J Clin Gastroenterol. 2016;50 Suppl 1:S4-5.

[19] Kałużna-Czaplińska J, Socha E, Rynkowski J. B vitamin supplementation reduces excretion of urinary dicarboxylic acids in autistic children. Nutr Res. 2011;31(7):497-502.

[20] Puig-Alcaraz C, Fuentes-Albero M, Cauli O. Relationship between adipic acid concentration and the core symptoms of autism spectrum disorders. Psychiatry Res. 2016;242:39-45.

[21] Duran M, Beemer FA, Tibosch AS, Bruinvis L, Ketting D, Wadman SK. Inherited 3-methylglutaconic aciduria in two brothers-another defect of leucine metabolism. J Pediatr. 1982;101(4):551-4. 
[22] Destefano F, Bhasin TK, Thompson WW, Yeargin-Allsopp M, Boyle C. Age at first measles-mumps-rubella vaccination in children with autism and school-matched control subjects: a population-based study in metropolitan Atlanta. Pediatrics. 2004;113(2):259-66.

[23] Gabriele S, Sacco R, Altieri L, Neri C, Urbani A, Bravaccio C, et al. Slow intestinal transit contributes to elevate urinary p-cresol level in Italian autistic children. Autism Res. 2015;9(7):752-9.

[24] Kałużna-Czaplińska J. Noninvasive urinary organic acids test to assess biochemical and nutritional individuality in autistic children. Clin Biochem. 2011a;44(8-9):686-91.

[25] Frye RE. Metabolic and mitochondrial disorders associated with epilepsy in children with autism spectrum disorder. Epilepsy Behav. 2015;47:147-57.

[26] Kałużna-Czaplińska J. Current applications of gas chromatography/mass spectrometry in the study of organic acids in urine. Crit Rev Anal Chem. 2011b;41(2):114-23.

[27] Kałużna-Czaplińska J, Błaszczyk S. The level of arabinitol in autistic children after probiotic therapy. Nutrition. 2012;28(2):124-6.

[28] Wishart DS, Jewison T, Guo AC, Wilson M, Knox C, Liu Y, et al. HMDB 3.0-The Human Metabolome Database in 2013. Nucleic Acids Res. 2013;41(Database issue):D801-7.

[29] Guneral F, Bachmann C. Age-related reference values for urinary organic acids in a healthy Turkish pediatric population. Clin Chem. 1994;40(6):862-8.

[30] Fuller R. Probiotics: Their Development and Use. In Probiotics: prospects of use in opportunistic infections. Ed. Fuller R, Heidt PJ, Rusch V, van der Waaij D. Herborn-Dill: Old Herborn University Seminar Monograph 1995;8:1-8.

[31] Ford AC, Quigley EM, Lacy BE, Lembo AJ, Saito YA, Schiller LR, et al. Efficacy of prebiotics, probiotics, and synbiotics in irritable bowel syndrome and chronic idiopathic constipation: systematic review and meta-analysis. Am J Gastroenterol. 2014;109(10):1547-61.

[32] Wasilewska J, Jarocka-Cyrta E, Kaczmarski M. Patogeneza zaburzeń przewodu pokarmowego u dzieci z autyzmem. Pol Merkuriusz Lek. 2009;27(157):40-3.
[33] Horvath K, Papadimitriou JC, Rabsztyn A, Drachenberg C, Tildon JT. Gastrointestinal abnormalities in children with autistic disorder. J Pediatr. 1999(5);35:559-63.

[34] Torrente F, Ashwood P, Day R, Machado N, Furlano RI, Anthony $A$, et al. Small intestinal enteropathy with epithelial IgG and complement deposition in children with regressive autism. Mol Psychiatry. 2002;7(4):375-82.

[35] de Magistris L, Familiari V, Pascotto A, Sapone A, Frolli $A$, lardino $P$, et al. Alterations of the intestinal barrier in patients with autism spectrum disorders and in their first-degree relatives. J Pediatr Gastroenterol Nutr. 2010;51(4):418-24.

[36] Yap IK, Angley M, Veselkov KA, Holmes E, Lindon JC, Nicholson JK. Urinary metabolic phenotyping differentiates children with autism, from their unaffected siblings and age-matched controls. J Proteome Res. 2010;9(6):2996-3004.

[37] Finegold SM, Molitoris D, Song Y, Liu C, Vaisanen ML, Bolte E, et al. Gastrointestinal microflora studies in late-onset autism. Clin Infect Dis. 2002;35(Suppl 1):S6-S16.

[38] Shattock P, Whiteley P. Biochemical aspects in autism spectrum disorders: updating the opioid-excess theory and presenting new opportunities for biomedical intervention. Expert Opin Ther Targets. 2002;6(2):175-83.

[39] World Health Organization. The ICD-10 classification of mental and behavioural disorders: diagnostic criteria for research. Geneva: World Health Organization; 2003.

[40] Zhang Q, Wang G, Du Y, Zhu L, Jiye A. GC/MS analysis of the rat urine for metabonomic research. J Chromatogr B Analyt Technol Biomed Life Sci. 2007;854(1-2):20-5.

[41] Kałużna-Czaplińska J, Żurawicz E, Struck W, Markuszewski M. Identification of organic acids as potential biomarkers in the urine of autistic children using gas chromatography/mass spectrometry. J Chromatogr B Analyt Technol Biomed Life Sci. 2014;966:70-6.

[42] Kuśmierek K, Głowacki R, Bald E. Analysis of urine for cysteine, cysteinylglycine, and homocysteine by high-performance liquid chromatography. Anal Bioanal Chem. 2006;385(5):855-60. 